\title{
Relative Orbit Determination for Unconnected Spacecraft within a Constellation
}

\author{
Tong Qin, ${ }^{\dagger}$ \\ Department of Precision Instrument, Tsinghua University, Beijing, 100084, China \\ Dong Qiao* \\ School of Aerospace Engineering, Beijing Institute of Technology, Beijing, 100081, China \\ Malcolm Macdonald \\ Department of Electronic and Electrical Engineering, University of Strathclyde, Glasgow, G1 1XJ, UK.
}

\section{I.Introduction}

LRGE constellations of spacecraft are set to become increasingly common. Orbit determination (OD) can be a non-trivial task for a large constellation operator, and efficient operations of such constellations is a crucial challenge. This Note aims at addressing one critical issue of large constellations, that is, the relative orbit determination (OD). Relative OD provides basic state information for subsequent spacecraft control and operation. In addition, determining relative orbits can be used to aid certain scientific and operational objectives, such as the gravity recovery in [1] and Earth observation in [2].

A significant volume of work [3-7] has focused on relative OD of two spacecraft, this is essentially an estimation problem based on inter-satellite ranging measurements. The prior work [8] has shown that inter-satellite ranging measurements are not enough to determine all orbit elements. Hill and Born [9-10] detail the obtainable orbit elements using inter-satellite range measurements, and that the full orbit element set can only be obtained through consideration of multi-body dynamics. Qin [11] further analyzed the sensitivity of relative orbit element determination to orbital geometric configurations, showing that the relative OD performance in some special configurations is degraded, making some orbit elements underivable.

To the best of the authors knowledge, no prior work has considered relative OD between non-directly connected spacecraft. The challenge of relative OD of unconnected spacecraft needs to be addressed so that every spacecraft can obtain its relative orbit, and the relative OD for a constellation can be completely derived. Without direct measurements, relative OD can neither be processed as an estimation problem nor a simple linear geometric problem.

\footnotetext{
${ }^{\dagger}$ Post doctor, Department of Precision Instrument. qintong0039@gmail.com

* Corresponding author, Professor, School of Aerospace Engineering, qiaodong@bit.edu.cn, 8610-68913488

* Professor, Department of Electronic and Electrical Engineering, malcolm.macdonald.102@ strath.ac.uk
} 
To realize relative OD for whole constellations, this Note first proposes an indirect relative OD method based on spherical geometry. A link between unconnected spacecraft is first established via an intermediate spacecraft. The relative orbit between the target and intermediate spacecraft is obtained using inter-satellite ranging measurements according to [11]. The indirect relative OD method is then developed based on spherical trigonometry to obtain the relative orbit of the target spacecraft. The obtainable orbit elements using this proposed method are analyzed in various geometric configurations, and for a different number of intermediated spacecraft. Then, the extension of the proposed method to orbits in perturbed dynamics is discussed.

\section{II.Indirect relative orbit determination method based on spherical geometry}

\section{A. Relative orbit determination models}

Consider two spacecraft $S_{A}$ and $S_{B}$ in, respectively, orbits $O_{A}$ and $O_{B}$ with the following Keplerian elements

$$
\begin{aligned}
& E_{A}=\left[a_{A}, e_{A}, i_{A}, \Omega_{A}, \omega_{A}, f_{A}\right] \\
& E_{B}=\left[a_{B}, e_{B}, i_{B}, \Omega_{B}, \omega_{B}, f_{B}\right] .
\end{aligned}
$$

where $a_{A}$ and $a_{B}$ are semi-major axes, $e_{A}$ and $e_{B}$ are eccentricities, $i_{A}$ and $i_{B}$ are true anomalies, $\Omega_{A}$ and $\Omega_{B}$ are longitude of ascending nodes, $\omega_{A}$ and $\omega_{B}$ are argument of periapsides, $f_{A}$ and $f_{B}$ are true anomalies, respectively. According to [11], the inter-satellite ranging measurement can at most determine the semi-major axis, eccentricity, relative orientation and true anomalies of two orbits, given by

$$
\boldsymbol{X}_{A, B}=\left[a_{A}, e_{A}, f_{A}, a_{B}, e_{B}, f_{B}, \theta, \phi_{A}, \phi_{B}\right]^{T} .
$$

where $\theta, \phi_{A}, \phi_{B}$ are three elements describing the relative orientation of two orbits projected on a celestial sphere, as shown in Fig.1. The angle $\theta$ is the angle between the two orbit planes, while $\phi_{A}$ and $\phi_{B}$ are the angular distances from the periapsis of $\mathrm{O}_{A}$ and $\mathrm{O}_{\mathrm{B}}$ to one of the two intersections of the orbits. This angle is positive in the direction of orbital motion.

The relative orientation elements can be expressed by the Keplerian elements, given by

$$
\begin{aligned}
& \theta=\cos ^{-1}\left(\cos \left(i_{A}\right) \cos \left(i_{B}\right)+\sin \left(i_{A}\right) \sin \left(i_{B}\right) \cos (\Delta \Omega)\right) \\
& \phi_{A}=\tan ^{-1}\left(\frac{\sin (\Delta \Omega)}{\sin \left(i_{A}\right) \cot \left(i_{B}\right)-\cos \left(i_{A}\right) \cos (\Delta \Omega)}\right)-\omega_{A}
\end{aligned}
$$




$$
\phi_{B}=\tan ^{-1}\left(\frac{\sin (\Delta \Omega)}{-\sin \left(i_{B}\right) \cot \left(i_{A}\right)+\cos \left(i_{B}\right) \cos (\Delta \Omega)}\right)-\omega_{B} .
$$

where $\Delta \Omega=\Omega_{2}-\Omega_{1}$ is the difference of longitude of ascending nodes.

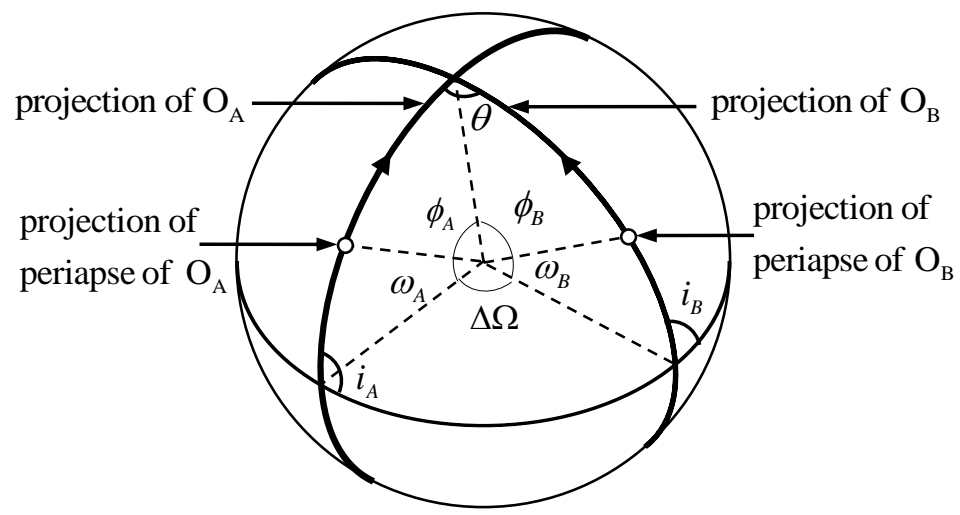

Fig.1 Relative orientation elements of two elliptic orbits projected onto a celestial sphere

\section{B. Indirect relative orbit determination methods}

In a constellation with more than two spacecraft, it is reasonable to consider that every spacecraft may not be linked to every other spacecraft. This might be due to the curvature of the Earth, due to limitations from the spacecraft hardware, or any other number of reasons. The conclusion in [11] is no longer applicable in determining the relative orbit between unconnected spacecraft. Therefore, an indirect method based on the spherical trigonometry is presented.

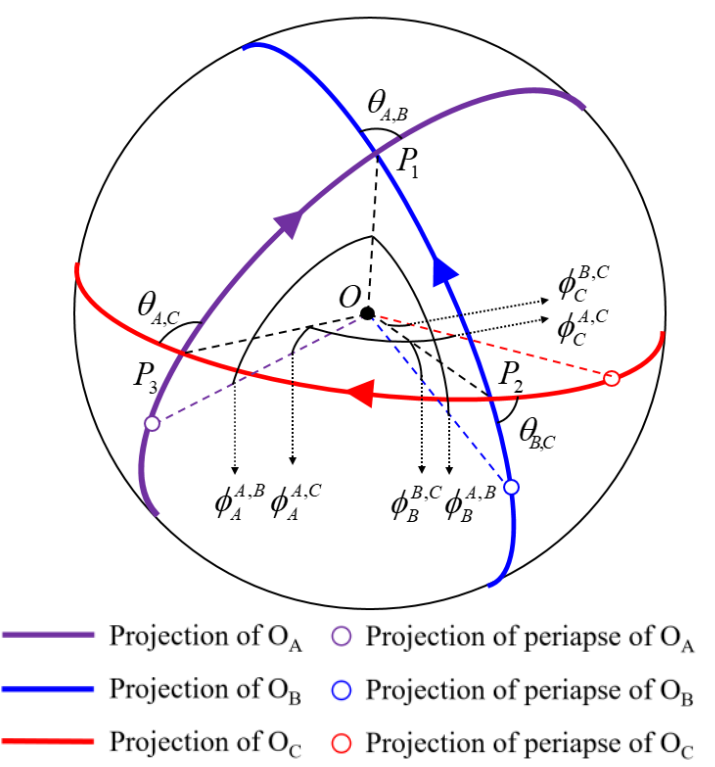

Fig. 2 Schematic of relative orientation of three orbits projected on a celestial sphere 
Consider a constellation with three spacecraft $\mathrm{S}_{\mathrm{A}}, \mathrm{S}_{\mathrm{B}}$, and $\mathrm{S}_{\mathrm{C}}$ with the corresponding orbits $\mathrm{O}_{\mathrm{A}}, \mathrm{O}_{\mathrm{B}}$, and $\mathrm{O}_{\mathrm{C}}$. The orbit projections on the celestial sphere are shown in Fig. 2. Crosslink is established between $\mathrm{S}_{\mathrm{A}}$ and $\mathrm{S}_{\mathrm{B}}$, and also between $\mathrm{S}_{\mathrm{B}}$ and $\mathrm{S}_{\mathrm{C}}$. Spacecraft $\mathrm{S}_{\mathrm{A}}$ and $\mathrm{S}_{\mathrm{C}}$ are not connected. Denote $\theta_{i, j}$ as the angle between great circles $i$ and $j$, and $\phi_{i}^{i, j}$ as the angular distance from the projection of periapse $i$ to the intersection of great circles $i$ and $j$. The indirect relative OD is to obtain the following state vector

$$
\boldsymbol{X}_{A, C}=\left[a_{A}, e_{A}, f_{A}, a_{C}, e_{C}, f_{C}, \theta_{A, C}, \phi_{A}^{A, C}, \phi_{B}^{B, C}\right]^{T} .
$$

Since $a_{A}, e_{A}$, and $f_{A}$ are determined via the inter-satellite ranging measurement between $\mathrm{S}_{\mathrm{A}}$ and $\mathrm{S}_{\mathrm{B}}$, and $a_{C}, e_{C}$, and $f_{C}$ are determined via the inter-satellite ranging measurement between $\mathrm{S}_{\mathrm{B}}$ and $\mathrm{S}_{\mathrm{C}}$, the indirect relative OD is to determine the relative orientation elements $\theta_{A, C}, \phi_{A}^{A, C}$, and $\phi_{C}^{A, C}$.

Based on the law of cosines of spherical trigonometry for angles, $\theta_{A, C}$ in Fig.3 is given by

$$
\theta_{A, C}=\cos ^{-1}\left(\cos \left(\theta_{A, B}\right) \cos \left(\theta_{B, C}\right)-\sin \left(\theta_{A, B}\right) \sin \left(\theta_{B, C}\right) \cos \left(\phi_{B}^{A, B}-\phi_{B}^{B, C}\right)\right)
$$

Obtaining $\theta_{A, C}$, the angular distances are calculated based on the law of sines, given as

$$
\begin{gathered}
\frac{\sin \left(\phi_{B}^{A, B}-\phi_{B}^{B, C}\right)}{\sin \left(\theta_{A, C}\right)}=\frac{\sin \left(\phi_{A}^{A, B}-\phi_{A}^{A, C}\right)}{\sin \left(\theta_{B, C}\right)}=\frac{\sin \left(\phi_{C}^{A, C}-\phi_{C}^{B, C}\right)}{\sin \left(\theta_{A, B}\right)} \\
\phi_{A}^{A, C}=\phi_{A}^{A, B}-\sin ^{-1}\left(\frac{\sin \left(\phi_{B}^{A, B}-\phi_{B}^{B, C}\right)}{\sin \left(\theta_{A, C}\right)} \sin \left(\theta_{B, C}\right)\right) \\
\phi_{C}^{A, C}=\phi_{C}^{B, C}+\sin ^{-1}\left(\frac{\sin \left(\phi_{B}^{A, B}-\phi_{B}^{B, C}\right)}{\sin \left(\theta_{A, C}\right)} \sin \left(\theta_{A, B}\right)\right) .
\end{gathered}
$$

Eqs. (8)-(11) give the process to calculate the relative orbit orientations of $\mathrm{O}_{\mathrm{A}}$ and $\mathrm{O}_{\mathrm{C}}$ in Fig.3. However, various relative geometric configurations can be constructed by the three orbits. Thus, the solutions of the relative orbit of two unconnected spacecraft is not unique. Considering the possible direction of the three orbits in a spherical triangular, there are in total eight types of geometrical configurations, as shown in Fig.3. 


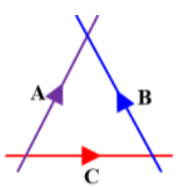

case 1

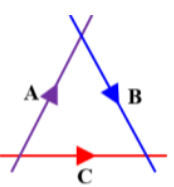

case 2

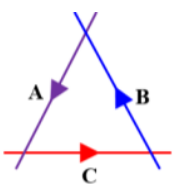

case 3

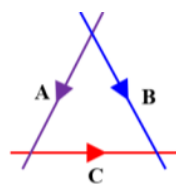

case 4

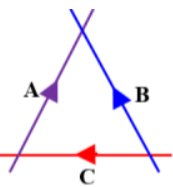

case 5

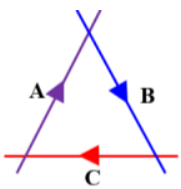

case 6

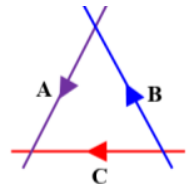

case 7

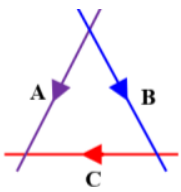

case 8

Fig. 3 All potentials of relative geometric configurations of three orbits in a spherical triangular

The computation equations for the relative orientation of $\mathrm{O}_{\mathrm{A}}$ and $\mathrm{O}_{\mathrm{c}}$ under various geometric configurations are given in table 1, accompanied with the classification criteria.

Table 1 Computation equations for the relative orientation of $\mathrm{O}_{\mathrm{A}}$ and $\mathrm{O}_{\mathrm{C}}$ under various configurations

\begin{tabular}{ccccc}
\hline \hline Case & Geometric criteria & $\theta_{A, C}$ & $\phi_{A}^{A, C}$ & $\phi_{C}^{A, C}$ \\
\hline 1 & $\phi_{A}^{A, B}>\phi_{A}^{A, C}, \phi_{B}^{A, B}>\phi_{B}^{B, C}, \phi_{C}^{B, C}>\phi_{C}^{A, C}$ & $\theta_{A, C}=\cos ^{-1}\left(\theta_{\cos }+\theta_{\text {sin }}\right)$ & $\phi_{A}^{A, C}=\phi_{A}^{A, B}-\Delta \phi_{A}$ & $\phi_{C}^{A, C}=\phi_{C}^{B, C}-\Delta \phi_{C}$ \\
2 & $\phi_{A}^{A, B}>\phi_{A}^{A, C}, \phi_{B}^{A, B}<\phi_{B}^{B, C}, \phi_{C}^{B, C}>\phi_{C}^{A, C}$ & $\theta_{A, C}=\cos ^{-1}\left(\theta_{\cos }+\theta_{\text {sin }}\right)$ & $\phi_{A}^{A, C}=\phi_{A}^{A, B}+\Delta \phi_{A}$ & $\phi_{C}^{A, C}=\phi_{C}^{B, C}+\Delta \phi_{C}$ \\
3 & $\phi_{A}^{A, B}<\phi_{A}^{A, C}, \phi_{B}^{A, B}>\phi_{B}^{B, C}, \phi_{C}^{B, C}>\phi_{C}^{A, C}$ & $\theta_{A, C}=\cos ^{-1}\left(\theta_{\cos }-\theta_{\sin }\right)$ & $\phi_{A}^{A, C}=\phi_{A}^{A, B}+\Delta \phi_{A}$ & $\phi_{C}^{A, C}=\phi_{C}^{B, C}-\Delta \phi_{C}$ \\
4 & $\phi_{A}^{A, B}<\phi_{A}^{A, C}, \phi_{B}^{A, B}<\phi_{B}^{B, C}, \phi_{C}^{B, C}>\phi_{C}^{A, C}$ & $\theta_{A, C}=\cos ^{-1}\left(\theta_{\cos }-\theta_{\text {sin }}\right)$ & $\phi_{A}^{A, C}=\phi_{A}^{A, B}-\Delta \phi_{A}$ & $\phi_{C}^{A, C}=\phi_{C}^{B, C}+\Delta \phi_{C}$ \\
5 & $\phi_{A}^{A, B}>\phi_{A}^{A, C}, \phi_{B}^{A, B}>\phi_{B}^{B, C}, \phi_{C}^{B, C}<\phi_{C}^{A, C}$ & $\theta_{A, C}=\cos ^{-1}\left(\theta_{\cos }-\theta_{\sin }\right)$ & $\phi_{A}^{A, C}=\phi_{A}^{A, B}-\Delta \phi_{A}$ & $\phi_{C}^{A, C}=\phi_{C}^{B, C}+\Delta \phi_{C}$ \\
6 & $\phi_{A}^{A, B}>\phi_{A}^{A, C}, \phi_{B}^{A, B}<\phi_{B}^{B, C}, \phi_{C}^{B, C}<\phi_{C}^{A, C}$ & $\theta_{A, C}=\cos ^{-1}\left(\theta_{\cos }-\theta_{\sin }\right)$ & $\phi_{A}^{A, C}=\phi_{A}^{A, B}+\Delta \phi_{A}$ & $\phi_{C}^{A, C}=\phi_{C}^{B, C}-\Delta \phi_{C}$ \\
7 & $\phi_{A}^{A, B}<\phi_{A}^{A, C}, \phi_{B}^{A, B}>\phi_{B}^{B, C}, \phi_{C}^{B, C}<\phi_{C}^{A, C}$ & $\theta_{A, C}=\cos ^{-1}\left(\theta_{\cos }+\theta_{\text {sin }}\right)$ & $\phi_{A}^{A, C}=\phi_{A}^{A, B}+\Delta \phi_{A}$ & $\phi_{C}^{A, C}=\phi_{C}^{B, C}+\Delta \phi_{C}$ \\
8 & $\phi_{A}^{A, B}<\phi_{A}^{A, C}, \phi_{B}^{A, B}<\phi_{B}^{B, C}, \phi_{C}^{B, C}<\phi_{C}^{A, C}$ & $\theta_{A, C}=\cos ^{-1}\left(\theta_{\cos }+\theta_{\text {sin }}\right)$ & $\phi_{A}^{A, C}=\phi_{A}^{A, B}-\Delta \phi_{A}$ & $\phi_{C}^{A, C}=\phi_{C}^{B, C}-\Delta \phi_{C}$ \\
\hline \hline
\end{tabular}

In table 1 ,

$$
\begin{gathered}
\theta_{\cos }=\cos \left(\theta_{A, B}\right) \cos \left(\theta_{B, C}\right), \\
\theta_{\text {sin }}=\sin \left(\theta_{A, B}\right) \sin \left(\theta_{B, C}\right) \cos \left(\phi_{B}^{A, B}-\phi_{B}^{B, C}\right), \\
\Delta \phi_{A}=\sin ^{-1}\left(\frac{\sin \left(\phi_{B}^{A, B}-\phi_{B}^{B, C}\right)}{\sin \left(\theta_{A, C}\right)} \sin \left(\theta_{B, C}\right)\right), \text { and } \\
\Delta \phi_{C}=\sin ^{-1}\left(\frac{\sin \left(\phi_{B}^{A, B}-\phi_{B}^{B, C}\right)}{\sin \left(\theta_{A, C}\right)} \sin \left(\theta_{A, B}\right)\right) .
\end{gathered}
$$

It is shown in Table 1 that the eight potential geometric configurations lead to four groups of computation equations. Cases (1) and (8) have the same solutions, as do cases (2) and (7), cases (3) and (6), and cases (4) and (5). It is therefore further considered whether the inputs of the indirect relative OD method can constrain the solution sets into uniqueness. Consider the three geometric configurations in Fig. 4. In Fig. 4(a), orbit $\mathrm{O}_{\mathrm{C}}$ is symmetric with the $\mathrm{O}_{\mathrm{C}}$ in Fig. 2 about $\mathrm{O}_{\mathrm{B}}$. In Fig. 4(b), orbit $\mathrm{O}_{\mathrm{A}}$ is symmetric with the $\mathrm{O}_{\mathrm{A}}$ in Fig.2 about $\mathrm{O}_{\mathrm{B}}$. In Fig.4 (c), orbits $\mathrm{O}_{\mathrm{A}}$ 
and $\mathrm{O}_{\mathrm{C}}$ are both symmetric with those in Fig. 2 about $\mathrm{O}_{\text {B }}$. Due to this symmetry, the four configurations in Fig.2 and Fig. 4 have the same relative orbit elements $\theta_{A, B}, \theta_{B, C}, \phi_{A}^{A, B}, \phi_{B}^{A, B}, \phi_{B}^{B, C}$, and $\phi_{C}^{B, C}$, which means the inputs of the relative OD method are the same in Fig.4 and Fig.2. But the relative geometry of $\mathrm{O}_{\mathrm{A}}$ and $\mathrm{O}_{\mathrm{C}}$ are different and the relative orientation elements are given in Table 2.

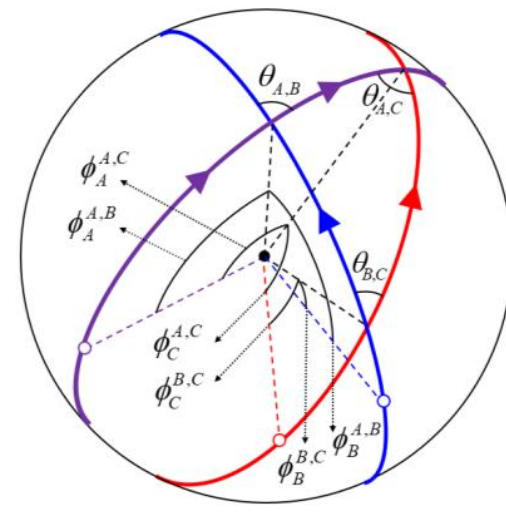

(a)

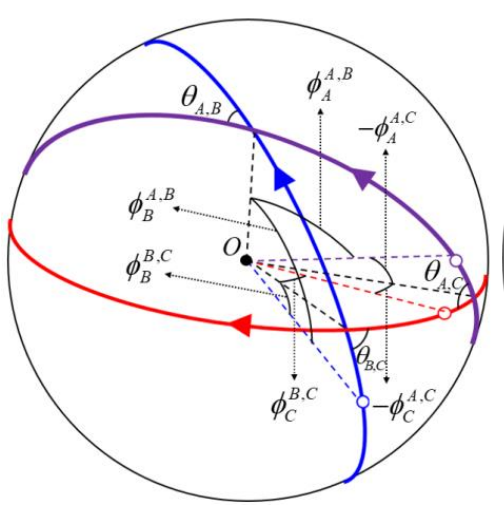

(b)

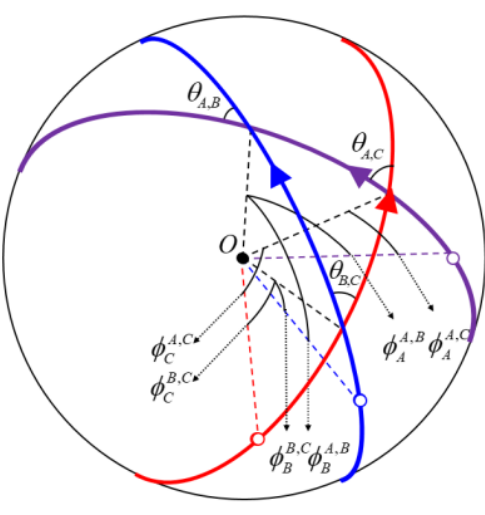

(c)

Fig.4 Three more potential configurations having the same relative orientation elements as Fig.2.

Table 2 Solutions for the relative orientation of $O_{\mathrm{A}}$ and $\mathrm{O}_{\mathrm{C}}$ under various geometric configurations

\begin{tabular}{|c|c|c|c|c|}
\hline Case & Geometric criteria & $\theta_{A, C}$ & $\phi_{A}^{A, C}$ & $\phi_{C}^{A, C}$ \\
\hline Fig.3 & $\phi_{A}^{A, B}>\phi_{A}^{A, C}, \phi_{B}^{A, B}>\phi_{B}^{B, C}, \phi_{C}^{B, C}<\phi_{C}^{A, C}$ & $\theta_{A, C}=\cos ^{-1}\left(\theta_{\cos }-\theta_{\text {sin }}\right)$ & $\phi_{A}^{A, C}=\phi_{A}^{A, B}-\Delta \phi_{A}$ & $\phi_{C}^{A, C}=\phi_{C}^{B, C}+\Delta \phi_{C}$ \\
\hline Fig.4(a) & $\phi_{A}^{A, B}<\phi_{A}^{A, C}, \phi_{B}^{A, B}>\phi_{B}^{B, C}, \phi_{C}^{B, C}<\phi_{C}^{A, C}$ & $\theta_{A, C}=\cos ^{-1}\left(\theta_{\cos }+\theta_{\sin }\right)$ & $\phi_{A}^{A, C}=\phi_{A}^{A, B}+\Delta \phi_{A}$ & $\phi_{C}^{A, C}=\phi_{C}^{B, C}+\Delta \phi_{C}$ \\
\hline Fig.4(b) & $\phi_{A}^{A, B}>\phi_{A}^{A, C}, \phi_{B}^{A, B}>\phi_{B}^{B, C}, \phi_{C}^{B, C}>\phi_{C}^{A, C}$ & $\theta_{A, C}=\cos ^{-1}\left(\theta_{\cos }+\theta_{\text {sin }}\right)$ & $\phi_{A}^{A, C}=\phi_{A}^{A, B}-\Delta \phi_{A}$ & $\phi_{C}^{A, C}=\phi_{C}^{B, C}-\Delta \phi_{C}$ \\
\hline Fig.4(c) & $\phi_{A}^{A, B}>\phi_{A}^{A, C}, \phi_{B}^{A, B}>\phi_{B}^{B, C}, \phi_{C}^{B, C}<\phi_{C}^{A, C}$ & $\theta_{A, C}=\cos ^{-1}\left(\theta_{\cos }-\theta_{\text {sin }}\right)$ & $\phi_{A}^{A, C}=\phi_{A}^{A, B}-\Delta \phi_{A}$ & $\phi_{C}^{A, C}=\phi_{C}^{B, C}+\Delta \phi_{C}$ \\
\hline
\end{tabular}

Considering the above four cases, there are three solution sets for the relative orientation of $\mathrm{O}_{\mathrm{A}}$ and $\mathrm{O}_{\mathrm{C}}$. Therefore, due to the symmetry of the relative orbits, the intermediate relative orientations cannot limit the solutions into uniqueness. An a priori value of each orbit is needed to distinguish the geometric configuration. Usually, each spacecraft roughly knows its orbit either from the ground station or onboard orbit integration. The rough orbit can help determine the geometric configurations, and the accurate relative orientation elements are obtained by the indirect relative OD method.

Note that the relative orientation elements determined from inter-satellite ranging measurements are the basic inputs of the geometry-based indirect relative OD method. The prior work of the authors illustrates that the obtainable relative elements are quite different under different orbital geometric configurations ${ }^{[11]}$, which means the inputs of the indirect relative OD method may change with configuration. 


\section{Sensitivity analysis on geometric configurations}

\section{A. For relative OD of two connected spacecraft}

In [11], it was shown that coplanar, circular, and symmetric configurations lead to fewer obtainable orbit elements. The relative OD results of two connected spacecraft in special geometric configurations are summarized in Table 3 and Table 4. The general geometric configurations refer to two non-coplanar, non-symmetric, and noncircular orbits.

Table 3 Obtainable relative orbit elements under non-coplanar geometric configurations

\begin{tabular}{ccc}
\hline \hline Case & Geometry description & Obtainable elements \\
\hline 1 & General geometric configuration & $a_{A}, e_{A}, a_{B}, e_{B}, f_{A}, f_{B}, \phi_{A}^{A, B}, \phi_{B}^{A, B}, \theta_{A, B}$ \\
2 & Non-coplanar circular orbit and elliptic orbit & $a_{A}, e_{A}, a_{B}, e_{B}, f_{A}-\phi_{A}^{A, B}, f_{B}, \phi_{B}^{A, B}, \theta_{A, B}$ \\
3 & Two non-coplanar circular orbits & $a_{A}, e_{A}, a_{B}, e_{B}, f_{A}-\phi_{A}^{A, B}, f_{B}-\phi_{B}^{A, B}, \theta_{A, B}$ \\
4 & Two symmetric elliptic orbits with the same phase & $a_{A}+a_{B}, e_{A}+e_{B}, f_{A}+f_{B}, \phi_{A}+\phi_{B}, \theta_{A, B}$ \\
5 & Two symmetric circular orbits with the same phase & $a_{A}+a_{B}, e_{A}+e_{B}, f_{A}+f_{B}-\phi_{A}-\phi_{B}, \theta_{A, B}$ \\
\hline \hline
\end{tabular}

Table 4 Obtainable relative orbit elements under coplanar geometric configurations

\begin{tabular}{ccc}
\hline \hline Case & Geometry description & Shape and Size \\
\hline 1 & Two different elliptic orbits & $a_{A}, e_{A}, a_{B}, e_{B}, f_{A}, f_{B}, \phi_{A}^{A, B}-\phi_{B}^{A, B}$ \\
2 & One circular orbit and one elliptic orbit & $a_{A}, e_{A}, a_{B}, e_{B}, f_{B}, f_{A}-\phi_{A}+\phi_{B}$ \\
3 & Two different circular orbits & $a_{A}, e_{A}, a_{B}, e_{B}, f_{A}-f_{B}$ \\
4 & The same elliptic orbit & $a_{A}, e_{A}, a_{B}, e_{B}, f_{A}, f_{B}, \phi_{A}^{A, B}-\phi_{B}^{A, B}$ \\
5 & The same circular orbit & $e_{A}, e_{B}, a_{A}-a_{B}, c_{1}\left(a_{A}+a_{B}\right)+2 c_{2}\left(f_{A}-f_{B}-\phi_{A}^{A, B}+\phi_{B}^{A, B}\right)$ \\
\hline \hline
\end{tabular}

It is seen in Table 3 and Table 4 that the coplanar configuration causes an unobservability of the angle between orbit planes. The circular orbit causes an unobservability of true anomaly and angular distance. There are even fewer obtainable elements in some severe geometric configurations. As the indirect relative OD method takes the elements obtained from inter-satellite ranging measurements as inputs, hence, a sensitivity analysis is performed to reveal the obtainable elements for unconnected spacecraft.

\section{B. For indirect relative OD of unconnected spacecraft}

\section{One intermediate spacecraft}

Without lose of generality, the geometric configuration in Fig.2 is taken as the example for sensitivity analysis. In Eq. (10), $\theta_{A, B}, \theta_{B, C}$ and $\phi_{B}^{A, B}-\phi_{B}^{B, C}$ are needed to calculate $\theta_{A, C}$. Based on the analysis in [11], the requirements for 
the geometric configuration to obtain these intermediate parameters are that, $\mathrm{O}_{\mathrm{A}}$ and $\mathrm{O}_{\mathrm{B}}$ are non-coplanar and nonsymmetric, and that $\mathrm{O}_{\mathrm{B}}$ and $\mathrm{O}_{\mathrm{C}}$ are non-coplanar and non-symmetric.

For the relative OD based on inter-satellite range data, the angle between orbit planes cannot be determined in coplanar geometry. However, this problem does not exist in the indirect relative OD method. When $\mathrm{O}_{\mathrm{A}}$ and $\mathrm{O}_{\mathrm{C}}$ are coplanar, it can be obtained from the inter-satellite range measurements that

$$
\theta_{A, B}=\theta_{B, C}, \phi_{B}^{A, B}=\phi_{B}^{B, C}
$$

Therefore, according to Eq. (10), it can be obtained that

$$
\theta_{A, C}=0
$$

Eq. (17) indicates that, even though the two orbits are coplanar, the angles between the two orbit planes can be determined if the intermediated spacecraft is non-coplanar with the two spacecraft. This point is of significance for designing the crosslink in a spacecraft constellation with multiple orbit planes to obtain the maximum relative elements. As long as there are no coplanar crosslinks, the angles between any two orbit planes can be determined.

In cases where $\theta_{A, C} \neq 0$, the determination of $\phi_{A}^{A, C}$ and $\phi_{C}^{A, C}$ depends on whether $\phi_{A}^{A, B}$ and $\phi_{C}^{B, C}$ can be obtained. In detail, if $\mathrm{O}_{\mathrm{A}}$ is an elliptic orbit, according to Table $3, \phi_{A}^{A, B}$ can be determined from the inter-satellite range measurements, and thus $\phi_{A}^{A, C}$ can be determined according to Eq. (12). If orbit $\mathrm{O}_{\mathrm{A}}$ is a circular orbit, only $f_{A}-\phi_{A}^{A, B}$ can be obtained, and then $f_{A}-\phi_{A}^{A, C}$ is solved.

In cases where $\theta_{A, C}=0$, which means $\mathrm{O}_{\mathrm{A}}$ and $\mathrm{O}_{\mathrm{C}}$ are coplanar, it satisfies the conditions $\theta_{A, B}=\theta_{B, C}$ and $\phi_{B}^{A, B}=\phi_{B}^{B, C}$. According to the right equality in Eq. (11), we get $\phi_{A}^{A, B}-\phi_{A}^{A, C}=\phi_{C}^{B, C}-\phi_{C}^{A, C}$. If $\phi_{A}^{A, B}$ and $\phi_{C}^{B, C}$ are both determined, which requires that $\mathrm{O}_{\mathrm{A}}$ and $\mathrm{O}_{\mathrm{C}}$ are both elliptic orbits, $\phi_{A}^{A, C}-\phi_{C}^{A, C}$ can be calculated. If one of them is a circular orbit, only the linear combination of true anomaly and angular distance can be obtained. The obtainable relative orientation elements between $\mathrm{O}_{\mathrm{A}}$ and $\mathrm{O}_{\mathrm{C}}$ are summarized in Table 5 .

Table 5. Obtainable relative orientation elements of $\mathrm{OA}$ and $\mathrm{OC}$ under various geometric configurations

\begin{tabular}{cc}
\hline \hline Relative configurations of $\mathrm{O}_{\mathrm{A}}$ and $\mathrm{O}_{\mathrm{C}}$ & Relative orientation elements \\
\hline Non-coplanar elliptic orbits & $\theta_{A, C}, \phi_{A}^{A, C}, \phi_{C}^{A, C}$ \\
Non-coplanar circular $\mathrm{O}_{\mathrm{A}}$ and elliptic orbit $\mathrm{O}_{\mathrm{C}}$ & $\theta_{A, C}, f_{A}-\phi_{A}^{A, C}, \phi_{C}^{A, C}$ \\
Non-coplanar circular $\mathrm{O}_{\mathrm{C}}$ and elliptic orbit $\mathrm{O}_{\mathrm{A}}$ & $\theta_{A, C}, \phi_{A}^{A, C}, f_{C}-\phi_{C}^{A, C}$
\end{tabular}


Non-coplanar circular orbits

Coplanar elliptic orbits

Coplanar circular orbits

$$
\begin{gathered}
\theta_{A, C}, f_{A}-\phi_{A}^{A, C}, f_{C}-\phi_{C}^{A, C} \\
\theta_{A, C}, \phi_{A}^{A, C}-\phi_{C}^{A, C} \\
\theta_{A, C},\left(f_{A}-\phi_{A}^{A, C}\right)-\left(f_{C}-\phi_{C}^{A, C}\right)
\end{gathered}
$$

Comparing with the direct relative OD via inter-satellite range measurements, the indirect relative OD has two advantages. One is the availability of the angle between orbit planes in coplanar geometry. The other is the availability of $\left(f_{A}-\phi_{A}^{A, C}\right)-\left(f_{C}-\phi_{C}^{A, C}\right)$ for two spacecraft in the same circular orbit. Therefore, the indirect relative OD method weakens sensitivity to geometric configurations, and, when there's a third spacecraft, the relative orbit elements of two orbit may be better determined.

\section{Two or more intermediate spacecraft}

When there are two or more intermediate spacecraft, the required two groups of intermediate relative orientation elements cannot be obtained via inter-satellite range measurements simultaneously. Assume spacecraft $\mathrm{S}_{\mathrm{D}}$ is linked to spacecraft $\mathrm{S}_{\mathrm{C}}$. When performing the relative OD of $\mathrm{S}_{\mathrm{A}}$ and $\mathrm{S}_{\mathrm{D}}$, the intermediate relative orientation elements of $\mathrm{S}_{\mathrm{A}}$ and $\mathrm{S}_{\mathrm{C}}$ need to be obtained via the indirect relative OD method. The relative orientations of $\mathrm{O}_{\mathrm{A}}$ and $\mathrm{O}_{\mathrm{D}}$ under a certain geometric configuration are given by

$$
\begin{gathered}
\theta_{A, D}=\cos ^{-1}\left(\cos \left(\theta_{A, C}\right) \cos \left(\theta_{C, D}\right)+\sin \left(\theta_{A, C}\right) \sin \left(\theta_{C, D}\right) \cos \left(\phi_{C}^{C, D}-\phi_{C}^{A, C}\right)\right) \\
\frac{\sin \left(\phi_{C}^{C, D}-\phi_{C}^{A, C}\right)}{\sin \left(\theta_{A, D}\right)}=\frac{\sin \left(\phi_{D}^{A, D}-\phi_{D}^{C, D}\right)}{\sin \left(\theta_{A, C}\right)}=\frac{\sin \left(\phi_{A}^{A, D}-\phi_{A}^{A, C}\right)}{\sin \left(\theta_{C, D}\right)} \\
\phi_{A}^{A, D}=\phi_{A}^{A, C}+\sin ^{-1}\left(\frac{\sin \left(\phi_{C}^{C, D}-\phi_{C}^{A, C}\right)}{\sin \left(\theta_{A, D}\right)} \sin \left(\theta_{C, D}\right)\right) \\
\phi_{D}^{A, D}=\phi_{D}^{C, D}+\sin ^{-1}\left(\frac{\sin \left(\phi_{C}^{C, D}-\phi_{C}^{A, C}\right)}{\sin \left(\theta_{A, D}\right)} \sin \left(\theta_{A, C}\right)\right) .
\end{gathered}
$$

To obtain the angle $\theta_{A, D}, \mathrm{O}_{\mathrm{C}}$ and $\mathrm{O}_{\mathrm{D}}$ still need to be non-coplanar and non-symmetric so that $\theta_{C, D}$ and $\phi_{C}^{C, D}$ (or $f_{C}-\phi_{C}^{C, D}$ if $\mathrm{O}_{\mathrm{C}}$ is circular) can be obtained. If the relative geometry between $\mathrm{O}_{\mathrm{A}}$ and $\mathrm{O}_{\mathrm{C}}$ is non-coplanar, $\phi_{C}^{A, C}$ (or $f_{C}-\phi_{C}^{A, C}$ if orbit $\mathrm{C}$ is circular) can be obtained from Table 3. Then, combining the intermediated state $\phi_{C}^{C, D}$ (or $f_{C}-\phi_{C}^{C, D}$ if orbit $\mathrm{C}$ is circular), $\phi_{C}^{A, C}-\phi_{C}^{C, D}$ are solved. If $\mathrm{O}_{\mathrm{A}}$ and $\mathrm{O}_{\mathrm{C}}$ are coplanar, it has $\theta_{A, C}=0$. Thus Eq. (18) can 
be simplified as

$$
\theta_{A, D}=\theta_{C, D}
$$

The angle $\theta_{A, D}$ is still solvable. Therefore, the determination of $\theta_{A, D}$ has no requirement for the relative geometric configuration between $\mathrm{O}_{\mathrm{A}}$ and $\mathrm{O}_{\mathrm{C}}$. Similar to the case with one intermediate spacecraft, the determination of angular distances $\phi_{A}^{A, D}$ and $\phi_{D}^{A, D}$ is also related to the eccentricities of $\mathrm{O}_{\mathrm{A}}$ and $\mathrm{O}_{\mathrm{D}}$. The detained analysis is omitted here.

The obtainable relative orientation elements of $\mathrm{O}_{\mathrm{A}}$ and $\mathrm{O}_{\mathrm{D}}$ are summarized in Table 6. Compared with the results in Table 5, the types of obtainable relative orientation elements of $\mathrm{O}_{A}$ and $\mathrm{O}_{D}$ are identical to those of $\mathrm{O}_{A}$ and $\mathrm{O}_{C}$. $\mathrm{By}$ parity of reasoning, the same relative orientation elements can be obtained when there are more than two intermediate spacecraft, illustrating the feasibility of the indirect relative OD method.

Table 6. Obtainable relative orientation elements of $\mathrm{OA}$ and OD

\begin{tabular}{cc}
\hline \hline Relative configurations of $\mathrm{O}_{\mathrm{A}}$ and $\mathrm{O}_{\mathrm{D}}$ & Relative orientation elements \\
\hline Non-coplanar elliptic orbits & $\theta_{A, D}, \phi_{A}^{A, D}, \phi_{D}^{A, D}$ \\
Non-coplanar circular $\mathrm{O}_{\mathrm{A}}$ and elliptic orbit $\mathrm{O}_{\mathrm{D}}$ & $\theta_{A, D}, f_{A}-\phi_{A}^{A, D}, \phi_{D}^{A, D}$ \\
Non-coplanar circular orbit $\mathrm{O}_{\mathrm{D}}$ and elliptic $\mathrm{O}_{\mathrm{A}}$ & $\theta_{A, D}, \phi_{A}^{A, D}, f_{D}-\phi_{D}^{A, D}$ \\
Non-coplanar circular orbits & $\theta_{A, D}, f_{A}-\phi_{A}^{A, D}, f_{D}-\phi_{D}^{A, D}$ \\
Coplanar elliptic orbits & $\theta_{A, D}, \phi_{A}^{A, D}-\phi_{D}^{A, D}$ \\
Coplanar circular orbits & $\theta_{A, D},\left(f_{A}-\phi_{A}^{A, D}\right)-\left(f_{D}-\phi_{D}^{A, D}\right)$ \\
\hline \hline
\end{tabular}

\section{Extension to Non-Keplerian Orbits}

The relative OD in two-body dynamics is the most difficult, or the most unobservable. The rotation of the constellation along three axes of the inertial coordinate system cannot be determined using inter-satellite range measurements ${ }^{[8]}$. Thus, the relative OD in two-body dynamics is taken as the general case to be investigated. Perturbed dynamics would improve the observability, and more orbit elements become observable. Therefore, once the direct and indirect relative OD can be realized in two-body dynamics, they can also be realized in perturbed dynamics more easily. Next, the extension technique to non-Keplerian orbits perturbed by non-spherical gravity is discussed.

Considering $\mathbf{J}_{2}$ perturbation, the gravity acceleration is rotationally symmetric along $\mathrm{z}$-axis of the inertial coordinate system. Using inter-satellite range measurements, only the longitude of ascending node is unobservable. Compared with relative OD in two-body dynamics, two more orbit elements (inclination and argument of periapsis) 
become observable. Therefore, $\Delta \Omega$. is enough to express the relative orbit of two spacecraft. The state model of relative OD is given by

$$
\boldsymbol{X}=\left[a_{A}, e_{A}, i_{A}, \omega_{A}, f_{A}, a_{B}, e_{B}, i_{B}, \omega_{B}, f_{B}, \Delta \Omega\right]
$$

The drift rate of each state in Eq. (23) is referred in [12]. The observation model in [11] is used, given by:

$$
\rho=\left\|\boldsymbol{r}_{B}-\boldsymbol{T}_{B}^{A} \boldsymbol{r}_{A}\right\|
$$

where $\boldsymbol{T}_{B}^{A}$ is the transformation matrix from orbit reference system of $\mathrm{O}_{\mathrm{A}}$ to $\mathrm{O}_{\mathrm{B}}$, and $r_{B}$ and $r_{A}$ are, in their own orbit reference systems, the position vectors of two spacecraft.

$$
\begin{aligned}
& \boldsymbol{r}_{A}=\left[r_{A} \cos \left(f_{A}\right), r_{A} \sin \left(f_{A}\right), 0\right]^{T} \\
& \boldsymbol{r}_{B}=\left[r_{B} \cos \left(f_{B}\right), r_{B} \sin \left(f_{B}\right), 0\right]^{T} \\
& r_{A}=\frac{a_{A}\left(1-e_{A}^{2}\right)}{1+e_{A} \cos \left(f_{A}\right)} \\
& r_{B}=\frac{a_{B}\left(1-e_{B}^{2}\right)}{1+e_{B} \cos \left(f_{B}\right)} \\
& \boldsymbol{T}_{B}^{A}=\left[\begin{array}{ccc}
\cos \left(\phi_{B}\right) & -\sin \left(\phi_{B}\right) & 0 \\
\sin \left(\phi_{B}\right) & \cos \left(\phi_{B}\right) & 0 \\
0 & 0 & 1
\end{array}\right]\left[\begin{array}{ccc}
1 & 0 & 0 \\
0 & \cos (\theta) & \sin (\theta) \\
0 & -\sin (\theta) & \cos (\theta)
\end{array}\right]\left[\begin{array}{ccc}
\cos \left(\phi_{A}\right) & \sin \left(\phi_{A}\right) & 0 \\
-\sin \left(\phi_{A}\right) & \cos \left(\phi_{A}\right) & 0 \\
0 & 0 & 1
\end{array}\right] .
\end{aligned}
$$

Use the measurement in Eq. (24) to estimate the states in Eq. (23). After obtaining the relative longitude of ascending node of two connected spacecraft, the indirect relative orbit of unconnected spacecraft can be obtained in an easier way, given by

$$
\Delta \Omega_{A, C}=\Delta \Omega_{A, B}+\Delta \Omega_{B, C} \text { or } \Delta \Omega_{A, C}=\Delta \Omega_{A, B}-\Delta \Omega_{B, C} .
$$

An a priori value of each orbit is also needed to distinguish the geometric configuration. Based on the analysis above, the indirect relative OD in perturbed dynamics can be taken as a special case of the situations in Section II. The numerical verification of this part is given in section V.C.

\section{V.Simulation}

An Earth-centered two-body dynamics simulation is applied to verify the theoretical analysis. The reference orbits 
are all integrated by the fourth-order Runge-Kutta method. The effect of the relative OD method in weakening the sensitivity on geometric configuration is studied via simulations in some special configurations. Then the relative OD in perturbed dynamics is simulated.

\section{A. Validation of indirect relative orbit determination method}

The orbit elements of a constellation of six spacecraft, on six elliptical orbits with a range of ascending node values is listed in Table 7; denoting the $i^{\text {th }}$ spacecraft as $\mathrm{S}_{\mathrm{i}}$ and the corresponding orbit as $\mathrm{O}_{\mathrm{i}}$. The projection of the six orbits on the celestial sphere is shown in Fig. 5, where $\mathrm{P}_{1}$ to $\mathrm{P}_{10}$ are ten intersection points. The crosslink is only established between $S_{i}$ and $S_{i+1}$ where $i=1,2,3,4$, 5. In the constellation, each spacecraft maintains a line-of-sight to its neighboring spacecraft, and as such the crosslink is always available.

Table 7 Orbit elements of the elliptic constellation

\begin{tabular}{ccccccc}
\hline \hline Spacecraft & $a(\mathrm{~km})$ & $e$ & $i(\mathrm{deg})$ & $\Omega(\mathrm{deg})$ & $\omega(\mathrm{deg})$ & $f(\mathrm{deg})$ \\
\hline $\mathrm{S}_{1}$ & 26560 & 0.3 & 60 & 20 & 10 & 20 \\
$\mathrm{~S}_{2}$ & 26560 & 0.3 & 60 & 80 & 10 & 80 \\
$\mathrm{~S}_{3}$ & 26560 & 0.3 & 60 & 140 & 10 & 140 \\
$\mathrm{~S}_{4}$ & 26560 & 0.3 & 60 & 200 & 10 & 200 \\
$\mathrm{~S}_{5}$ & 26560 & 0.3 & 60 & 260 & 10 & 260 \\
$\mathrm{~S}_{6}$ & 26560 & 0.3 & 60 & 320 & 10 & 320 \\
\hline \hline
\end{tabular}

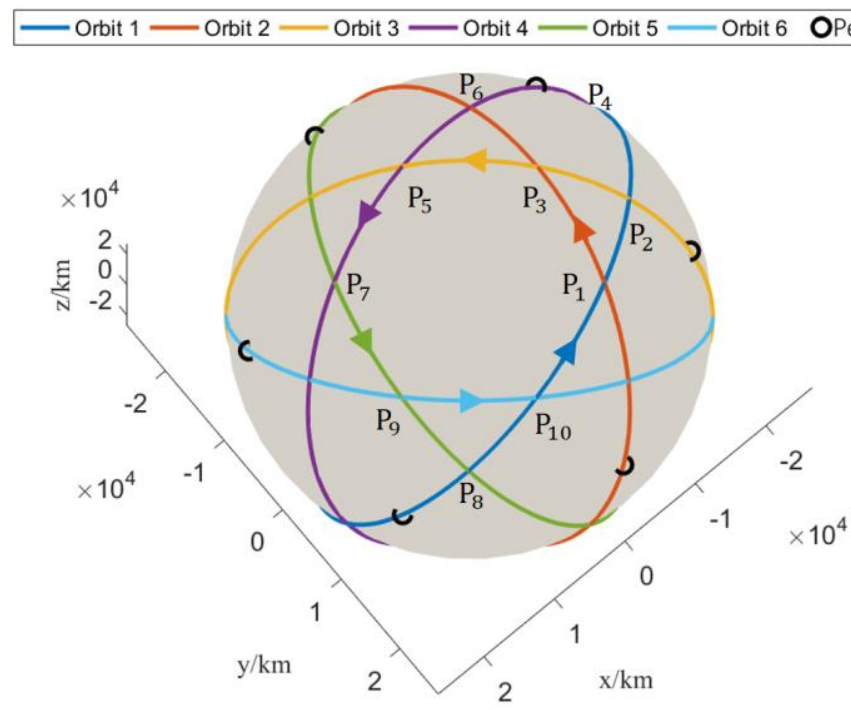

Fig. 5 Projection of the elliptic constellation on a celestial spherical

The relative orientation elements between $\mathrm{O}_{1}$ and $\mathrm{O}_{3}, \mathrm{O}_{1}$ and $\mathrm{O}_{4}, \mathrm{O}_{1}$ and $\mathrm{O}_{5}, \mathrm{O}_{1}$ and $\mathrm{O}_{6}$ are calculated to validate the indirect relative OD method. The initial knowledge errors of semi-major axis, eccentricity, inclination, longitude of ascending node, argument of periapsis, and true anomaly are, respectively, $10 \mathrm{~km}, 0.01,0.1^{\circ}, 0.1^{\circ}, 0.1^{\circ}, 0.1^{\circ}$. Inter- 
satellite range measurements with an accuracy of $1 \mathrm{~m}$ are used to estimate the relative orbits of connected spacecraft. The estimated relative orientation elements of unconnected spacecraft are computed based on the analysis in section II. According to the geometry in Fig. 5, the spherical triangles used to obtain the relative orientation are shown in Fig. 6.

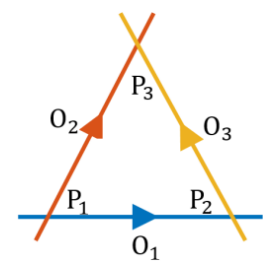

(a) $\mathrm{O}_{1}$ and $\mathrm{O}_{3}$

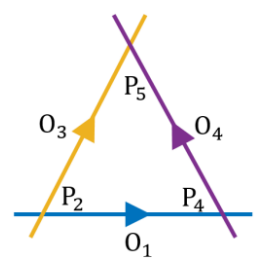

(b) $\mathrm{O}_{1}$ and $\mathrm{O}_{4}$

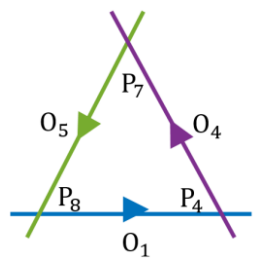

(c) $\mathrm{O}_{1}$ and $\mathrm{O}_{5}$

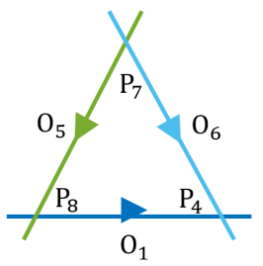

(d) $\mathrm{O}_{1}$ and $\mathrm{O}_{6}$

Fig. 6 Spherical triangles used to calculate the relative orientation elements of various pairs of orbits

Fig.6(a)-(d) correspond to various geometric configurations between the target spacecraft and intermediate spacecraft. The sequential estimation of the relative orbit between connected spacecraft over time is conducted according to the model in [11]. The relative orbit of unconnected spacecraft is determined according to the analysis in section II. The relative orientation errors of unconnected spacecraft are shown in Fig.7. It is shown that the orientation errors between estimated and real relative orientation elements of unconnected spacecraft all converge, indicating that the indirect relative OD method succeeds in obtaining the relative orbit for spacecraft without a direct link.
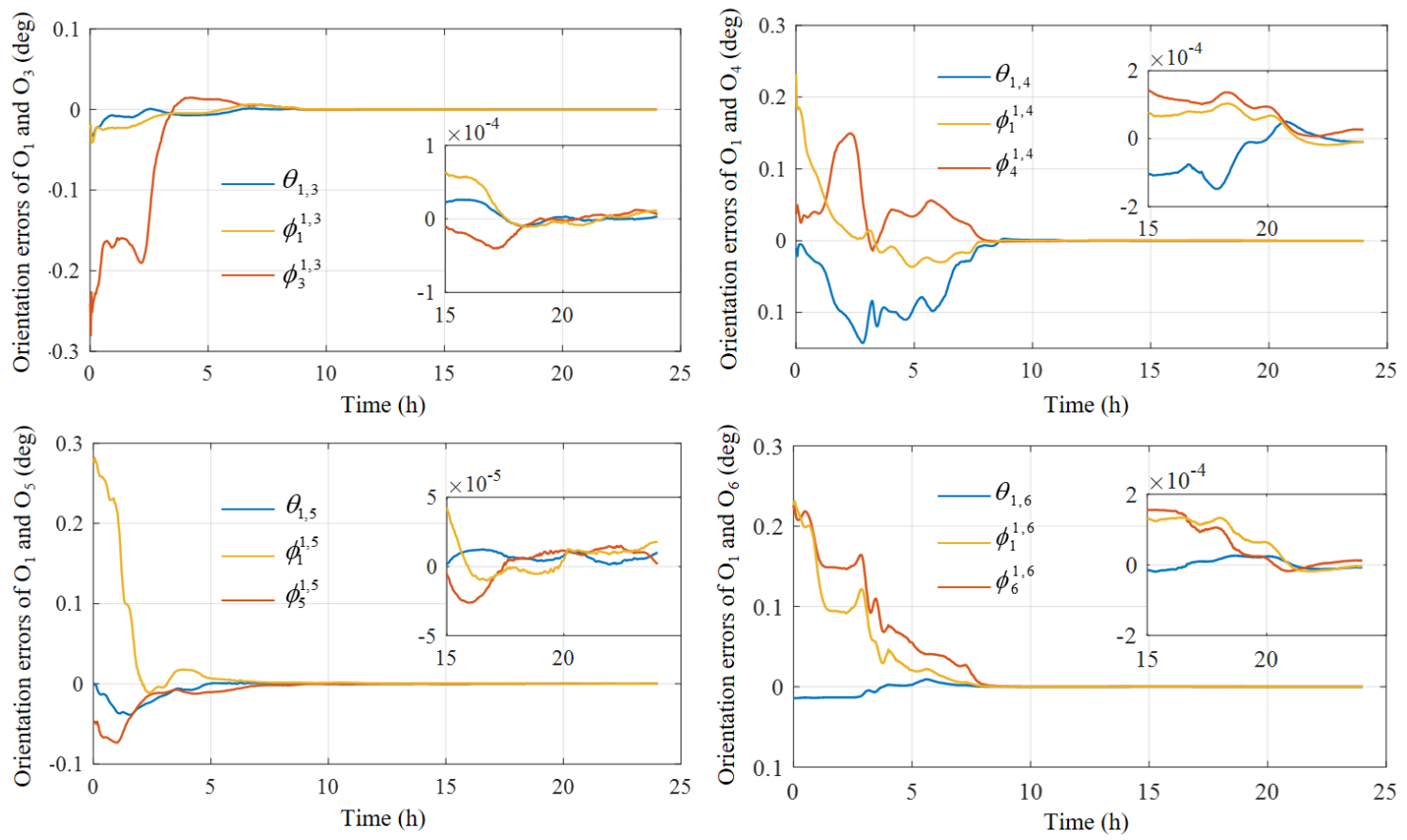

Fig. 7 Errors between estimated and real relative orientation elements of unconnected spacecraft 


\section{B. Promotion in sensitivity}

The indirect relative OD approach indicates that the surface angle of two coplanar orbits can be obtained via a non-coplanar intermediate spacecraft. Consider three spacecraft, among which two move in the same orbit and the other in a non-coplanar orbit; such orbit elements are shown in Table 8.

Table 8 Orbit elements of two coplanar orbits and one non-coplanar orbit

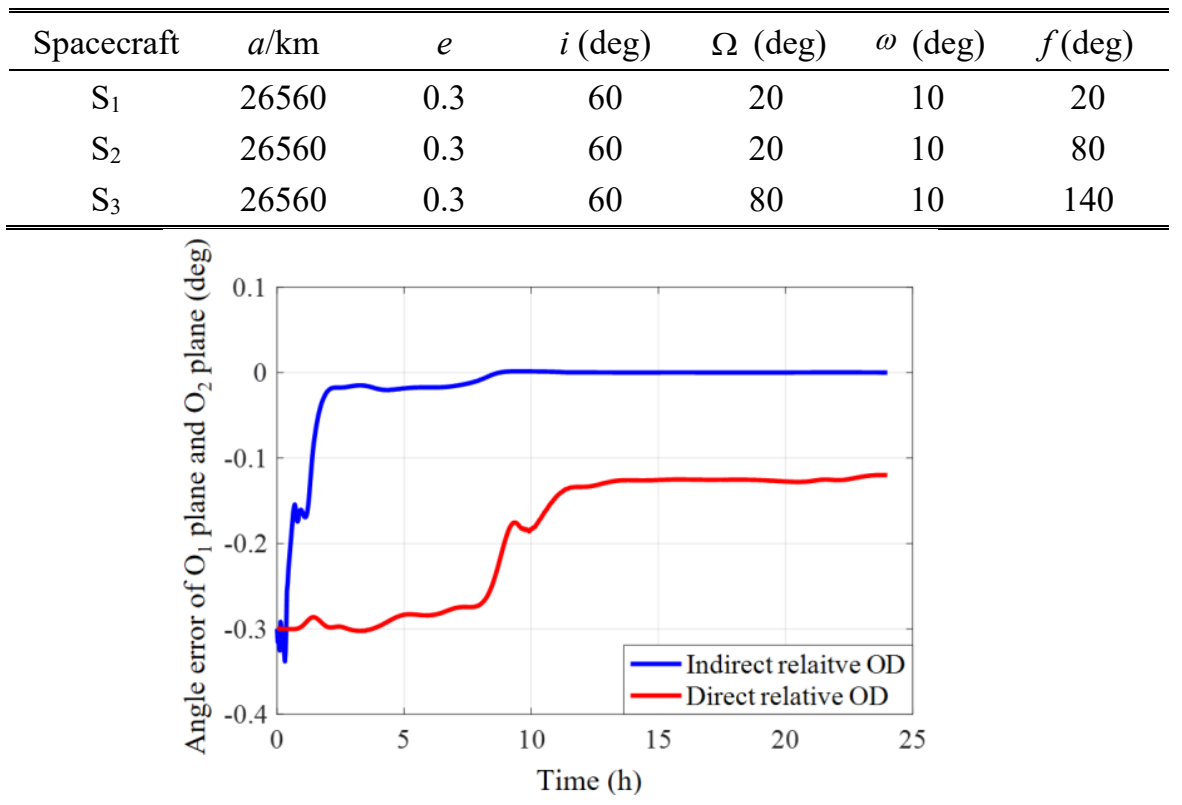

Fig. 8 Error of angle of two coplanar orbit planes using different OD methods

The angle errors between $\mathrm{O}_{1}$ and $\mathrm{O}_{2}$ obtained by indirect and direct relative OD method are shown in Fig. 8 . According to [11], the coplanar geometry leads to the unobservability of the angle of orbit planes. Thus, the direct relative OD error fails to converge. Involving a non-coplanar spacecraft and taking it as an intermediate spacecraft, the surface angle of the two coplanar spacecraft can be accurately computed via the indirect relative OD approach.

If both the eccentricities of $\mathrm{O}_{1}$ and $\mathrm{O}_{2}$ become zero, according to case 5 in Table 4, the direct relative OD using inter-satellite range measurements between $\mathrm{S}_{1}$ and $\mathrm{S}_{2}$ will reduce observability, and the semi-major axis cannot be determined. With an elliptic Orbit $\mathrm{O}_{3}$ in the constellation, $\mathrm{S}_{1}$ and $\mathrm{S}_{2}$ can determine their semi-major axis through relative ranging to $S_{3}$, as shown in Fig. 9 .
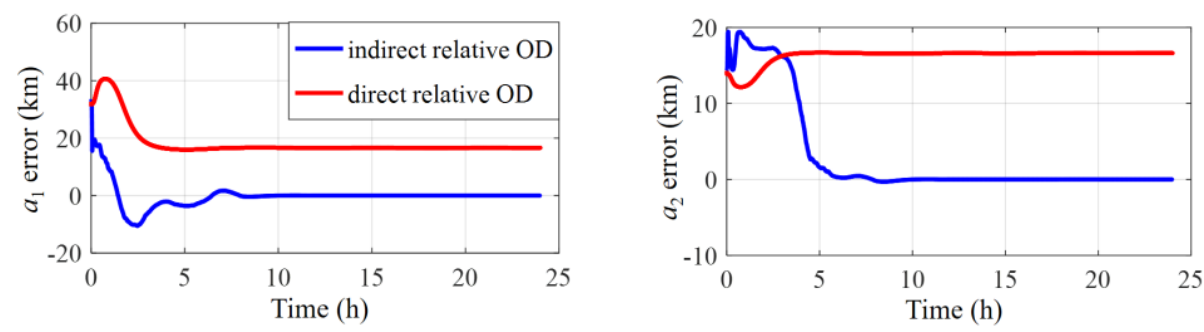

Fig. 9 Comparison of semi-major axis error using indirect relative OD and direct relative OD method 


\section{Extension to non-Keplerian orbits}

The orbit elements of spacecraft $S_{1}$ and $S_{2}$ in Table 7 are estimated to validate the relative OD in perturbed dynamics. It is shown in Fig. 10 that only the longitude of ascending node cannot be determined when considering $\mathbf{J}_{2}$ perturbation. The errors of $\Delta \Omega$ converge to near zero, illustrating that $\Delta \Omega$ can be used to express the relative orientation of two orbits in perturbed dynamics. Obtaining the relative orientation of connected spacecraft via intersatellite range measurements, the relative orientation of unconnected spacecraft can be obtained according to Eq. (30).
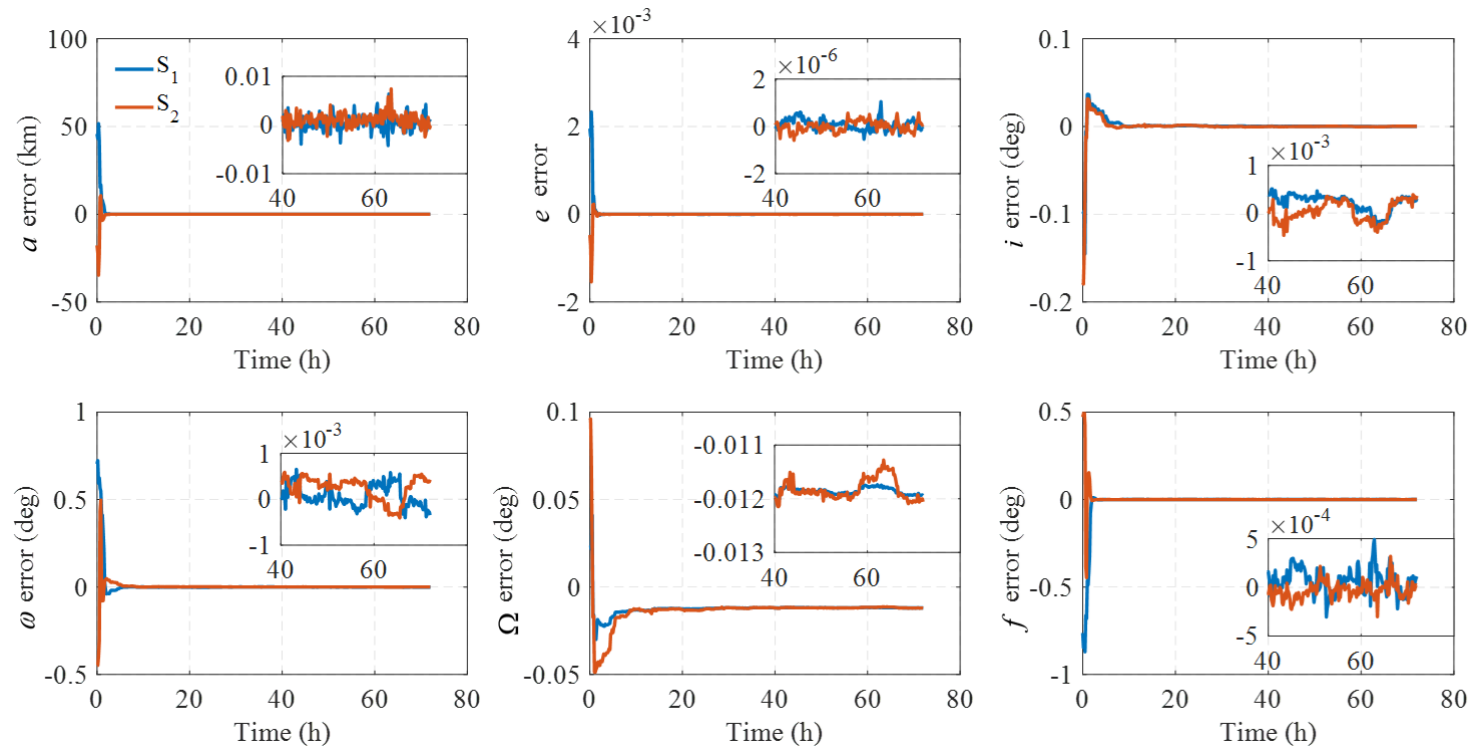

(a) Orbit element errors of $\mathrm{S}_{1}$ and $\mathrm{S}_{2}$

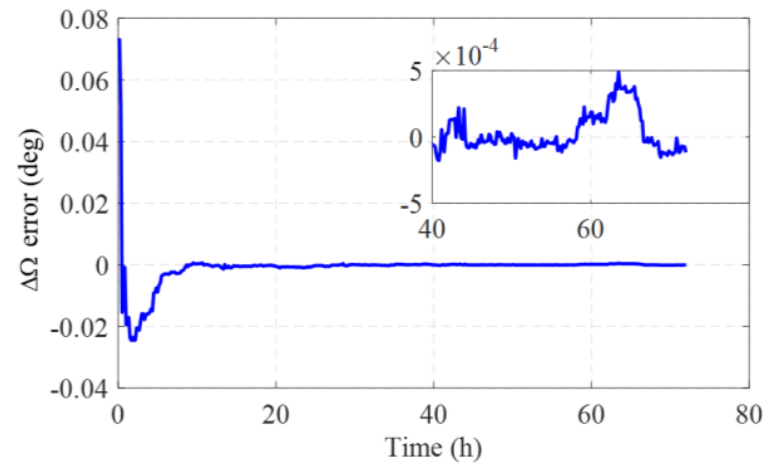

(b) Relative orientation error

Fig.10 Orbit determination errors in $\mathbf{J}_{2}$ perturbed dynamics using inter-satellite range measurements 


\section{VI.Conclusion}

Via intermediate spacecraft, the relative orientation elements of two unconnected orbits are determined by an indirect relative orbit determination method based on spherical geometry. Given the orientation elements with respect to the intermediate spacecraft, the relative orientation elements of the target spacecraft have three solution sets, and a priori orbit knowledge is needed to make the solution unique. Of special note is that, in a coplanar geometric configuration, the angle between two orbit planes can be determined using the indirect relative orbit determination method, which cannot be realized using direct orbit determination method. The indirect relative orbit determination approach for orbits in two-body dynamics can be also applied in perturbed dynamics where more orbit elements are observable.

\section{Acknowledgement}

This paper is sponsored by The National Natural Science Foundation of China (Grant No. 51827806).

\section{References}

[1] Mao, X., Visser, P., and van den IJssel, J., “Absolute and Relative Orbit Determination for the CHAMP/GRACE constellation,” Advances in Space Research, Vol. 63, No. 12, 2019, pp. 3816-3834.

https://doi.org/10.1016/j.asr.2019.02.030

[2] Lee, S., and Mortari, D., "Design of Constellations for Earth Observation with Intersatellite Links," Journal of Guidance, Control, and Dynamics, Vol. 4, No. 5, 2016, pp. 1-9.

https://doi.org/10.2514/1.G001710

[3] Abusali, P., Tapley, B. D., and Schutz. B. E, "Autonomous Navigation of Global Positioning System Satellites Using CrossLink Measurements,” Journal of Guidance, Control, and Dynamics, Vol. 21, No. 2, 1998, pp. 321-327.

https://doi.org/10.2514/2.4238

[4] Psiaki, M. L., “Autonomous Orbit Determination for two Spacecraft from Relative Position Measurements,” Journal of Guidance, Control, and Dynamics, Vol. 22, No. 2, 1999, pp. 305-312.

https://doi.org/10.2514/2.4379

[5] Gao, Y., Xu, B., and Zhang, L., "Feasibility Study of Autonomous Orbit Determination Using Only the Crosslink Range measurement for a Combined Navigation Constellation,” Chinese Journal of Aeronautics, Vol. 27, No. 5, 2014, pp. 11991210. 
https://doi.org/10.1016/j.cja.2014.09.005

[6] Butcher, E. A., and Wang, J., "On Kalman Filtering and Observability in Nonlinear Sequential Relative Orbit Estimation," Journal of Guidance, Control, and Dynamics, Vol. 40, No. 9, 2017, pp. 2167-2182.

https://doi.org/10.2514/1.G002702

[7] Yu, F., He, Z., and Xu, N., "Autonomous navigation for GPS using inter-satellite ranging and relative direction measurements," Acta Astronautica, Vol. 160, 2019, pp. 646-655.

https://doi.org/10.1016/j.actaastro.2019.03.011

[8] Liu, C., and Liu, L., “Orbit Determination Using Satellite-to-Satellite tracking Data," Chinese Journal of Astronomy and Astrophysics, Vol. 1, No. 3, 2001, pp. 281-286.

https://doi.org/10.1088/1009-9271/1/3/281

[9] Hill, K. A., and Born, G. H., “Autonomous Interplanetary Orbit Determination Using Satellite-to-Satellite tracking,” Journal of Guidance, Control, and Dynamics, Vol. 30, No. 3, 2007, pp. 679-686 https://doi.org/10.2514/2.3416

[10] Hill, K. A., and Born, G. H., “Autonomous Orbit Determination from Lunar halo Orbits Using Crosslink Range,” Journal of Spacecraft and Rockets, Vol. 45, No. 3, 2008, pp. 548-553.

https://doi.org/10.2514/1.32316

[11] Qin, T., Qiao, D., Macdonald, M., "Relative Orbit Determination Using only Intersatellite Range Measurements,” Journal of Guidance, Control, and Dynamics, Vol. 42, No. 3, 2019, pp. 703-710. https://doi.org/10.2514/1.G003819

[12] Hamel, J., Lafontaine, J., "Linearized Dynamics of Formation Flying Spacecraft on a $\mathbf{J}_{2}$-Perturbed Elliptical Orbit,” Journal of Guidance Control and Dynamics, Vol. 30, No. 6, 2007, pp. 1649-1658.

https://doi.org/10.2514/1.29438 